\title{
Performance Improvement of SONOS Device as UV Total Dose Nonvolatile Sensor with Bottom-silicon-rich and Top-nitrogen-rich Nitride Structure
}

\author{
Wen-Ching Hsieh $^{1 *}$ and Fuh-Cheng Jong ${ }^{2}$ \\ ${ }^{1}$ Department of Opto-Electronic System Engineering, Minghsin University of Science and Technology, \\ Xinxing Road 1, Xinfeng 30401, Taiwan \\ ${ }^{2}$ Electronic Engineering Department, Southern Taiwan University of Science and Technology, \\ 1 Nan-Tai Street, Yungkang District, Tainan 710, Taiwan
}

(Received April 16, 2018; accepted April 4, 2019)

Keywords: UV, sensor, SONOS, MOS, radiation

Silicon-silicon dioxide-silicon nitride-silicon dioxide-silicon (SONOS) capacitor devices with a bottom-silicon-rich and top-nitrogen-rich nitride bilayer stacked trapping layer (hereafter, SBNT-SONOS) have potential applications in UV total dose (TD) nonvolatile sensors. UV radiation induces a significant increase in the threshold voltage $V_{T}$ of SBNT-SONOS UV TD nonvolatile sensors. Experimental results indicate that the UV-induced increase in the $V_{T}$ of the SBNT-SONOS capacitor device under a positive gate voltage (PGV) is nearly $3 \mathrm{~V}$ after UV irradiation at $100 \mathrm{~mW} \cdot \mathrm{s} / \mathrm{cm}^{2}$ TD. The change in the $V_{T}$ of the SBNT-SONOS capacitor after UV irradiation is also dependent on UV TD of up to $100 \mathrm{~mW} \cdot \mathrm{s} / \mathrm{cm}^{2}$. The charge-retention loss of the nonvolatile SBNT-SONOS capacitor after 10-year retention is below $10 \%$. The UV TD information can be permanently stored and accumulated in nonvolatile SBNT-SONOS capacitor devices. Devices with a single standard nitride layer as the trapping layer show lower performance and poor reliability as UV TD sensors. Devices with a bottom-silicon-rich and top-nitrogen-rich nitride bilayer trapping layer show higher performance and better reliability as UV TD sensors than devices with a single standard nitride layer. The SBNT-SONOS capacitor device used in this study has demonstrated the feasibility of nonvolatile UV TD sensing.

\section{Introduction}

The measurement of UV radiation total dose (TD) is very important in various UV radiation applications, such as biotechnology and medical technology. Semiconductor dosimeters have many advantages. The dose-sensing areas of semiconductor dosimeters are very small, and their dose sensitivity can be high in a small constrained space. A silicon-silicon dioxide-silicon nitride-silicon dioxide-silicon (SONOS) capacitor device has been shown to be suitable for UV radiation TD nonvolatile sensor applications. ${ }^{(1-3)}$ UV radiation induces a significant increase in the threshold voltage $V_{T}$ of a SONOS capacitor device, and this UV-induced increase in $V_{T}$

*Corresponding author: e-mail: wchsieh@must.edu.tw

https://doi.org/10.18494/SAM.2019.2332 
correlates with UV TD. The reliability characteristic of $V_{T}$ retention in the SONOS capacitor device is good, even after 10-year retention. The UV TD information can be permanently stored and accumulated in this nonvolatile SONOS capacitor device. ${ }^{(1-6)}$

For UV TD data writing, UV radiation and positive gate voltage (PGV) were applied simultaneously to the SONOS capacitor device. Under UV irradiation and a PGV, negative charges are injected from the substrate into the charge-trapping layer of the nonvolatile SONOS capacitor device, where they are trapped. The build-up of negative charges changes the threshold voltage $V_{T}$, and the $V_{T}$ shift depends on the absorbed UV TD. As a result, negative charges are accumulated permanently in the trapping layers of the SONOS capacitor device. This is the UV-radiation-induced charging process in the nonvolatile SONOS capacitor device. ${ }^{(7,8)}$

In this paper, we propose a device with a bilayer nitride structure as the charge storage layer. This device shows improved performance and reliability as a UV TD sensor compared with a device with a single uniform nitride layer. A SONOS capacitor device with a bottomsilicon-rich and top-nitrogen-rich nitride as the bilayer stacked charge-trapping layer (hereafter, SBNT-SONOS) was fabricated in this study. The UV-radiation-induced charging effect and the charge-retention reliability of the SBNT-SONOS capacitor devices fabricated in this study were significantly improved. The electrical characteristics of the SBNT-SONOS capacitor devices under various UV TD conditions, including the UV radiation-induced charge effect, gate leakage current, and charge-retention reliability, are the main subjects of this study. Figure 1(a) shows a cross-sectional view of a SONOS device.

\section{Experimental Details}

Four types of SONOS device were prepared by adjusting the $\mathrm{Si}-\mathrm{N}$ composition ratio during nitride deposition for the SONOS capacitor in this study. SONOS capacitor structures were fabricated on a p-type-resistivity $15-25 \mathrm{ohm}-\mathrm{cm} \mathrm{Si} \ll 100 »$ substrate. We used thermal $\mathrm{SiO}_{2}$ for the tunneling oxide layer, nitride $\mathrm{Si}_{3} \mathrm{~N}_{4}$ for the trapping layer prepared by chemical vapor deposition (CVD), tetraethyloxysilane (TEOS) $\left[\mathrm{Si}\left(\mathrm{OC}_{2} \mathrm{H}_{5}\right)_{4}\right]$ for the blocking $\mathrm{SiO}_{2}$ prepared by CVD on the gate dielectric, and polysilicon for the gate material prepared by low-pressure

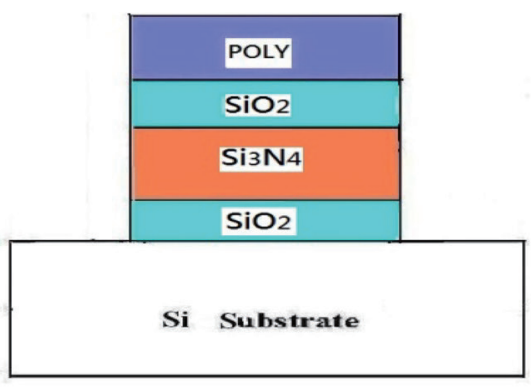

(a)

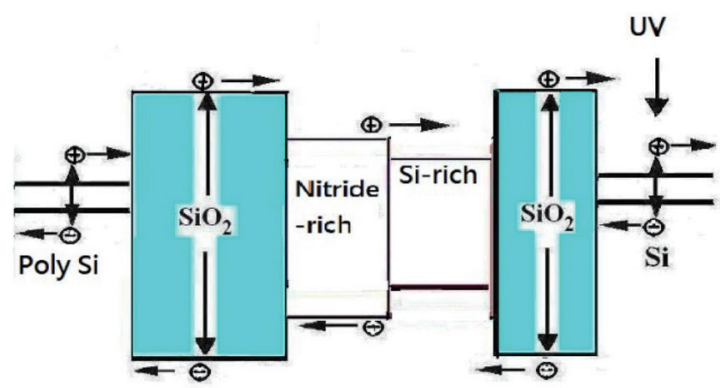

(b)

Fig. 1. (Color online) (a) Cross-sectional view of a SONOS capacitor device and (b) charge generation and trapping in a SBNT-SONOS capacitor after UV irradiation. 
chemical vapor deposition (LPCVD) . The tunneling $\mathrm{SiO}_{2}$ was formed on the wafers using an advanced clustered vertical furnace. After the tunneling oxide was formed, silicon nitride $\left(\mathrm{Si}_{3} \mathrm{~N}_{4}\right.$; hereafter, nitride) was formed as the charge-trapping layer by LPCVD on the SONOS capacitor device. The following four types of SONOS device prepared by adjusting the $\mathrm{Si}-\mathrm{N}$ composition ratio during nitride deposition in this study by adjusting the gas flow rate ratio of $\mathrm{SiH}_{2} \mathrm{Cl}_{2}$ to $\mathrm{NH}_{3}$ during charge-trapping nitride film deposition are listed in Table 1: (1) a SONOS capacitor with standard nitride $\left(\mathrm{SiH}_{2} \mathrm{Cl}_{2}: \mathrm{NH}_{3}=0.25: 1\right)$ as the charge-trapping layer (hereafter, STDSONOS), (2) a SONOS capacitor with a single N-rich nitride layer $\left(\mathrm{SiH}_{2} \mathrm{Cl}_{2}: \mathrm{NH}_{3}=0.1: 1\right)$ as the charge-trapping layer (hereafter, N-SONOS), (3) a SONOS capacitor with a single Si-rich nitride layer $\left(\mathrm{SiH}_{2} \mathrm{Cl}_{2}: \mathrm{NH}_{3}=2: 1\right)$ as the charge-trapping layer (hereafter, Si-SONOS), and (4) a SONOS capacitor with bilayer stacked trapping layer of Si-rich nitride $\left(\mathrm{SiH}_{2} \mathrm{Cl}_{2}: \mathrm{NH}_{3}=2: 1\right)$ on the bottom and a $\mathrm{N}$-rich nitride layer $\left(\mathrm{SiH}_{2} \mathrm{Cl}_{2}: \mathrm{NH}_{3}=0.1: 1\right)$ on the top as the charge-trapping layer (hereafter, $\mathrm{SBNT}-\mathrm{SONOS}$ ). The $\mathrm{SiO}_{2}-\mathrm{Si}_{3} \mathrm{~N}_{4}-\mathrm{SiO}_{2}$ (hereafter, ONO) gate stack consisted

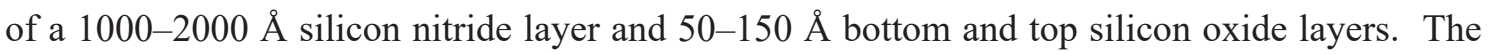
polysilicon (200-400 nm) used for the control gate was formed by LPCVD. For comparison, the four types of SONOS capacitor device had the same thicknesses of the tunneling oxide, trapping nitride, and blocking oxide layers.

Before UV TD data writing, a negative gate voltage (NGV) of $-40 \mathrm{~V}$ was first applied to the SONOS capacitor devices to "erase" the native charges in the ONO charge-trapping layer of these SONOS devices before UV irradiation. During UV TD data writing, UV radiation (UV LED; wavelength, $400 \mathrm{~nm}$ ) and a PGV were applied simultaneously to the SONOS capacitor devices. The various UV irradiation and PGV conditions are listed in Table 2. After UV TD data writing, $V_{T}$ was measured at room temperature using an HP4156A parameter analyzer. The experimental results of the gate capacitance at various applied gate voltages $\left(C_{G}-V_{G}\right)$ were obtained with a computer-controlled HP4284 parameter analyzer, and the $C_{G}-V_{G}$ curves were measured by sweeping $V_{G}$ at room temperature. Figure 1(b) shows the charge generation and trapping states of the gate dielectric in the SBNT-SONOS capacitor devices after UV irradiation.

Table 1

Various types of SONOS device with different charge-trapping layers.

\begin{tabular}{lc}
\hline SONOS & Charge-trapping layer \\
\hline STD & Standard nitride (single layer) \\
S & Si-rich nitride (single layer) \\
N & N-rich nitride (single layer) \\
SBNT & Si-rich nitride on bottom and N-rich nitride on top (bilayer) \\
\hline
\end{tabular}

Table 2

Various types of SBNT-SONOS device at various UV TDs and PGVs.

\begin{tabular}{lcc}
\hline SBNT-SONOS & $\mathrm{UV} \mathrm{TD}\left(\mathrm{mW} \cdot \mathrm{s} / \mathrm{cm}^{2}\right)$ & PGV $(\mathrm{V})$ \\
\hline U0V0 & 0 & 0 \\
U100V0 & 100 & 0 \\
U0V20 & 0 & 20 \\
U100V20 & 100 & 20 \\
\hline
\end{tabular}




\section{Results and Discussion}

\section{1 $\quad V_{T}$ shift in SBNT-SONOS after UV irradiation}

Figure 2 shows the $C_{G}-V_{G}$ curves for an SBNT-SONOS device before and after UV irradiation. The SBNT-SONOS capacitor device is initially in the erased state. An NGV $\left(V_{G}=\right.$ $-40 \mathrm{~V}$ ) was first applied to the SBNT-SONOS capacitor device to "erase" the native charges in the ONO charge-trapping layer. For UV TD data writing, UV irradiation and a PGV $\left(V_{G}=20\right.$ V) were applied simultaneously to the SBNT-SONOS capacitor device. As shown in Fig. 2, $V_{T}$ is about $-2 \mathrm{~V}$ for the SBNT-SONOS capacitor device before UV irradiation, and about $1 \mathrm{~V}$ for the device after UV irradiation at $100 \mathrm{~mW} \cdot \mathrm{s} / \mathrm{cm}^{2} \mathrm{TD}$ under a PGV $\left(V_{G}=20 \mathrm{~V}\right)$. As illustrated in Fig. 2, the CG-VG curve of SBNT-SONOS shifted to the right after UV irradiation at TD of $100 \mathrm{~mW} \cdot \mathrm{s} / \mathrm{cm}^{2}$ under the $\mathrm{PGV}$ of $20 \mathrm{~V}$. This indicates that $\mathrm{UV}$ radiation of $100 \mathrm{~mW} \cdot \mathrm{s} / \mathrm{cm}^{2} \mathrm{TD}$ induces an increase in $V_{T}$ of $3 \mathrm{~V}$ for the SBNT-SONOS capacitor under a PGV of $20 \mathrm{~V}$. This positive $V_{T}$ shift is in agreement with previous studies ${ }^{(1-3)}$ and is due to an increase in the net total amount of negative trapped charges accumulated in the ONO gate dielectric layer after UV irradiation. This UV radiation-induced positive $V_{T}$ shift in the UV-irradiated SBNT-SONOS device under PGV $20 \mathrm{~V}$ results from electron excitation by UV photons being injected over the $\mathrm{Si}-\mathrm{SiO}_{2}$ potential barrier into the trapping layer and finally being trapped in the nitride chargetrapping layer of ONO..$^{(1-3)}$

The UV radiation writing induces a significant increase in $V_{T}$ for the SBNT-SONOS capacitor devices. It is considered that this change in $V_{T}$ is mostly due to the significant increase in the amount of electrons trapped in the gate dielectric ONO after UV TD data writing. It is considered that the amount of electrons trapped in the gate insulator owing to UV TD data writing is greater than the amount of holes trapped in the gate dielectric ONO after UV TD data writing. The change in $V_{T}$ correlates with both the amount of trapped charges and the TD of UV radiation exposure. These trapped charges are accumulated in the gate dielectric layer, so the UV TD record cannot be destroyed or disturbed by UV TD data writing or reading. The UV TD data in the SONOS capacitor devices can be erased to the original null state by opposite-charge injection.

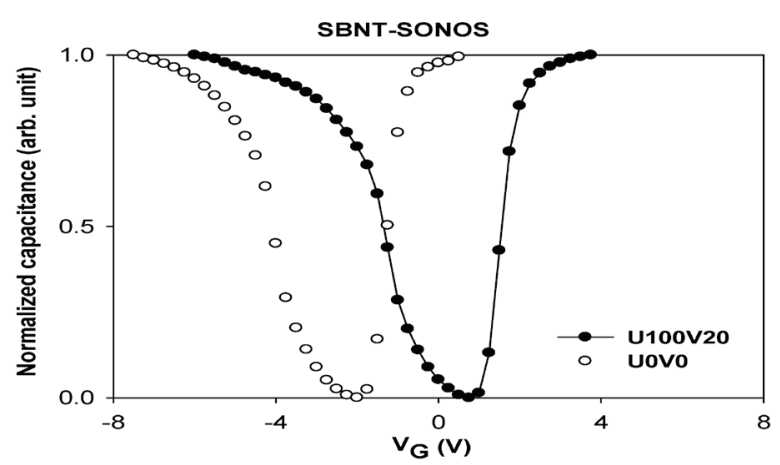

Fig. 2. $C_{G}-V_{G}$ curve for an SBNT-SONOS device before and after UV irradiation at TD of $100 \mathrm{~mW} \cdot \mathrm{s} / \mathrm{cm}^{2}$. 


\section{2 $\quad V_{T}$ increase vs UV TD in SBNT-SONOS capacitor after UV irradiation}

The increase in $V_{T}$ is plotted against the UV TD for SBNT-SONOS capacitors under the PGV of $20 \mathrm{~V}$ in Fig. 3(a). The increase correlates with both the increases in UV TD and the amount of negative charges trapped in the gate dielectric. However, $V_{T}$ increased more slowly when UV TD was larger than $30 \mathrm{~mW} \cdot \mathrm{s} / \mathrm{cm}^{2}$. The $V_{T}$ of the SBNT-SONOS capacitors changed markedly even at a low UV TD $\left(5 \mathrm{~mW} \cdot \mathrm{s} / \mathrm{cm}^{2}\right)$ under a high PGV $\left(V_{G}=20 \mathrm{~V}\right)$ and also changed negligibly even under a high gate PGV $\left(V_{G}=20 \mathrm{~V}\right)$ and zero UV TD $\left(0 \mathrm{~mW} \cdot \mathrm{s} / \mathrm{cm}^{2}\right)$. The experimental results of this study are in agreement with those of previous studies. ${ }^{(1-3)}$

Figure 3(b) shows the change in $V_{T}$ vs the PGV for an SBNT-SONOS capacitor device under a UV TD of $100 \mathrm{~mW} \cdot \mathrm{s} / \mathrm{cm}^{2}$. The $V_{T}$ shift induced at this UV TD for the SBNT-SONOS device was more obvious under a PGV of $20 \mathrm{~V}$ than that under a PGV of $10 \mathrm{~V}$, as shown in Fig. 3(b). Under a higher PGV, electrons were swept by a higher electric field and more electrons were captured by more charge-trapping centers of the ONO trapping layer. The $V_{T}$ of the SBNTSONOS capacitor was changed markedly under a high PGV $\left(V_{G}=20 \mathrm{~V}\right)$ even at a low UV $\mathrm{TD}\left(5 \mathrm{~mW} \cdot \mathrm{s} / \mathrm{cm}^{2}\right)$ [as shown in Fig. 3(a)], but the $V_{T}$ of the SBNT-SONOS capacitors was not changed significantly under a low PGV $\left(V_{G}=10 \mathrm{~V}\right)$ even at a high UV TD $\left(100 \mathrm{~mW} \cdot \mathrm{s} / \mathrm{cm}^{2}\right)$, as shown in Fig. 3(b). As illustrated in Figs. 3(a) and 3(b), the change in the $V_{T}$ of SONOS was ignorable under only UV irradiation (without a PGV), and also under only a PGV (without UV irradiation). It is considered that both UV radiation and a PGV must be applied to the SBNTSONOS capacitor device simultaneously to write UV radiation TD data, and that the significant increase in $V_{T}$ is due to a significant increase in the amount of electrons trapped in the gate dielectric ONO layer after simultaneous application UV radiation and PGV. The experimental results of this study are in agreement with those of previous studies. ${ }^{(1-3)}$

A comparison of changes in the $V_{T}$ of the four SONOS devices after UV irradiation at TD of $100 \mathrm{~mW} \cdot \mathrm{s} / \mathrm{cm}^{2}$ under a PGV of $20 \mathrm{~V}$ is shown in Fig. 4(a). A comparison of the relative charge amount for the four SONOS devices after UV irradiation at TD of $100 \mathrm{~mW} \cdot \mathrm{s} / \mathrm{cm}^{2}$ under a PGV of $20 \mathrm{~V}$ is shown in Fig. 4(b). To enable the comparison, the four SONOS capacitor devices had the same thicknesses of the tunneling oxide, trapping nitride, and blocking oxide

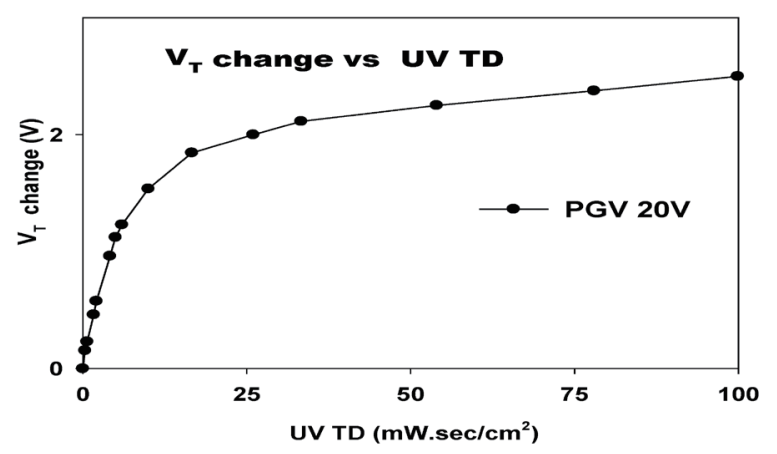

(a)

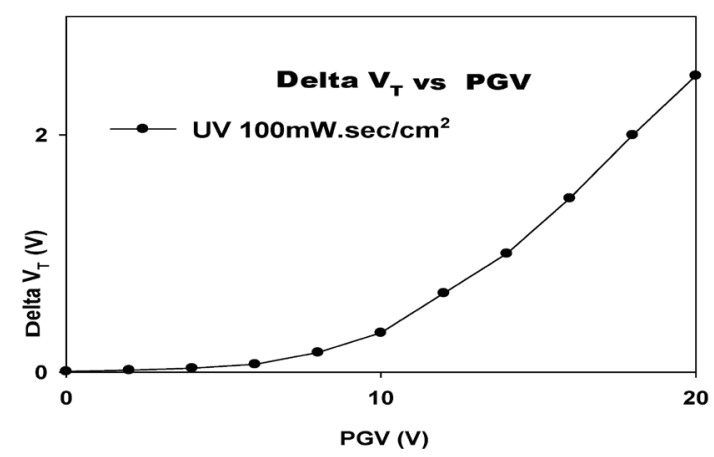

(b)

Fig. 3. (a) Dependence of increase in $V_{T}$ on UV radiation TD for an SBNT-SONOS under PGV of $20 \mathrm{~V}$. (b) Delta $V_{T}$ vs PGV for an SBNT-SONOS device after UV irradiation at TD of $100 \mathrm{~mW} \cdot \mathrm{s} / \mathrm{cm}^{2}$. 


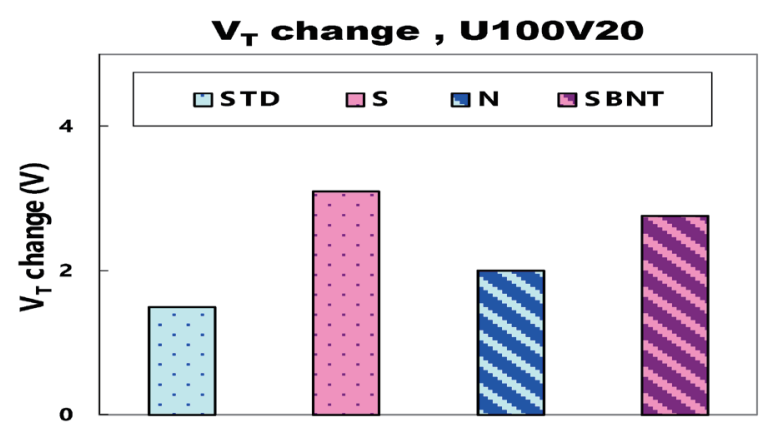

(a)

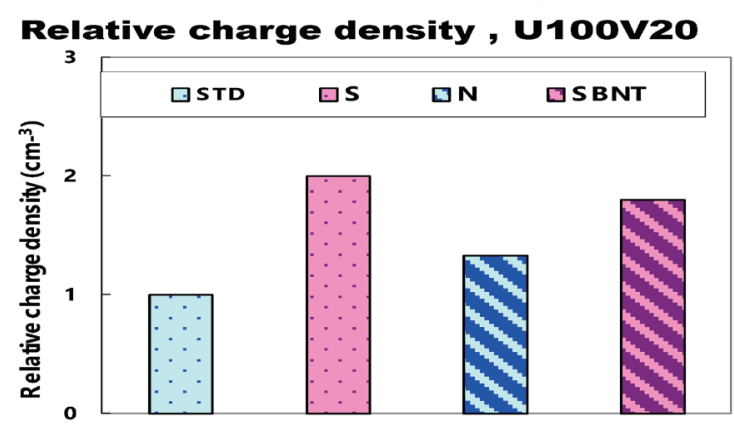

(b)

Fig. 4. (Color online) Comparisons of (a) the changes in $V_{T}$ and (b) the relative charge amount for the four SONOS devices after UV irradiation at TD of $100 \mathrm{~mW} \cdot \mathrm{s} / \mathrm{cm}^{2}$ under PGV of $20 \mathrm{~V}$.

layers. The radiation-induced change in charge amount was calculated from delta $V_{T}$ using the Terman method. ${ }^{(9)}$ The $V_{T}$ shift for N-SONOS was greater than that for STD-SONOS after UV irradiation as shown in Fig. 4(a), which results from the fact that the amount of UV radiationinduced charges trapped in the N-SONOS capacitor is greater than that in the STD-SONOS capacitor under a PGV. Note that the S-SONOS device with a single Si-rich nitride trapping layer demonstrates a larger $V_{T}$ shift than the N-SONOS device with a single N-rich nitride layer after UV irradiation as shown in Fig. 4(a). The Si-rich nitride trapping layer has a higher trap density and therefore shows better performance as a UV TD sensor than the N-rich nitride trapping layer. This result also agreed with previous studies of a Si-rich nitride charge-trapping layer in a MONOS device. ${ }^{(9,10)}$ Furthermore, the $V_{T}$ shift of SBNT-SONOS was greater than that of N-SONOS after UV irradiation as shown in Fig. 4(a). As shown above, the bilayer stacked trapping layer with Si-rich nitride on the bottom has a higher negative trap density and a larger amount of radiation-induced negative charges accumulated owing to UV irradiation, and therefore shows better performance as a UV TD sensor than a N-rich nitride. It is interesting to note that the UV radiation-induced increase in the $V_{T}$ of the SBNT-SONOS device was twice that of the STD-SONOS device after UV irradiation at $100 \mathrm{~mW} \cdot \mathrm{s} / \mathrm{cm}^{2}$ TD under a $20 \mathrm{~V}$ PGV. The increase in the radiation-induced $V_{T}$ shift in SBNT-SONOS can be explained by the increase in the amount of radiation-induced net negative charges in SBNT-SONOS compared with that in STD-MONOS.

\subsection{Measurement of gate leakage current}

Comparisons of the gate currents at $20 \mathrm{~V}$ PGV for the four types SONOS device before and after UV irradiation at $100 \mathrm{~mW} \cdot \mathrm{s} / \mathrm{cm}^{2} \mathrm{TD}$ under a $20 \mathrm{~V}$ PGV are respectively shown in Figs. 5(a) and 5(b). After UV irradiation at $100 \mathrm{~mW} \cdot \mathrm{s} / \mathrm{cm}^{2} \mathrm{TD}$ under a $20 \mathrm{~V} \mathrm{PGV}$, the gate current of the S-SONOS capacitor at a $20 \mathrm{~V}$ PGV increased more significantly than that of the STDSONOS capacitor, as can be seen in Figs. 5(a) and 5(b). The figures also show that a much shallower negative-charge trap energy level $E_{T A}$ is observed for S-SONOS than for STD- 


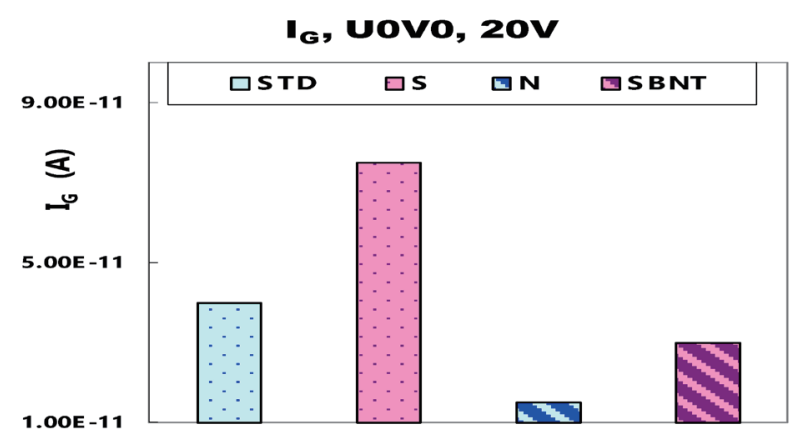

(a)

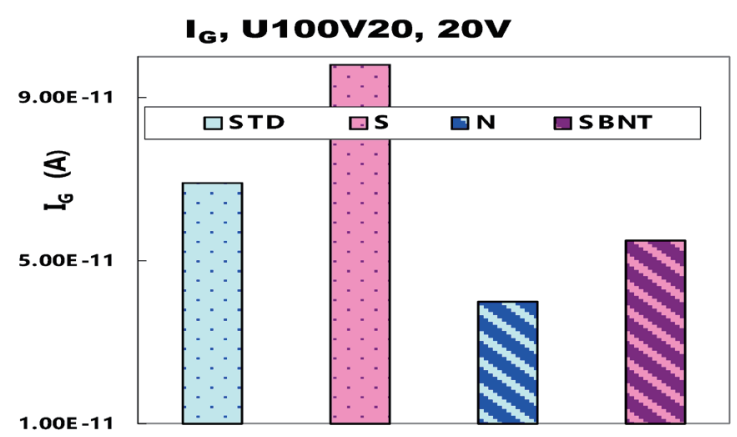

(b)

Fig. 5. (Color online) Comparisons of gate currents at PGV of $20 \mathrm{~V}$ for the four SONOS devices (a) before UV irradiation and (b) after UV irradiation at $100 \mathrm{~mW} \cdot \mathrm{s} / \mathrm{cm}^{2}$ TD under PGV of $20 \mathrm{~V}$.

MONOS. Relatively shallow negative-charge trap energy levels $E_{T A}$ originating from $\mathrm{Si}-\mathrm{H}$ or Si dangling bonds were observed for S-SONOS compared with STD-SONOS. This result also agreed with the previous studies of the Si-rich nitride charge-trapping layer in a MONOS device. $(9,10)$ In contrast, the N-SONOS capacitor showed better gate current leakage characteristics at a $20 \mathrm{~V}$ PGV than the STD-SONOS capacitor after UV irradiation at $100 \mathrm{~mW} \cdot \mathrm{s} / \mathrm{cm}^{2} \mathrm{TD}$ under a $20 \mathrm{~V}$ PGV [Figs. 5(a) and 5(b)]. A much deeper negative-charge $E_{T A}$ was observed for N-SONOS with the N-rich trapping layer than for STD-MONOS. This result also agreed with the previous studies of a MONOS device with the N-rich charge-trapping layer. ${ }^{(9,10)}$ As illustrated in Figs. 5(a)and 5(b), the gate oxide leakage current of the SBNT-SONOS capacitor device did not increase significantly after UV irradiation at $100 \mathrm{~mW} \cdot \mathrm{s} / \mathrm{cm}^{2} \mathrm{TD}$ under a PGV 20 V compared with STD-SONOS.

\section{4 $V_{T}$ stability vs retention time}

$V_{T}$ vs retention time for an SBNT-SONOS capacitor device before and after UV irradiation at $100 \mathrm{~mW} \cdot \mathrm{s} / \mathrm{cm}^{2}$ TD under a $20 \mathrm{~V} \mathrm{PGV}$ is illustrated in Figs. 6(a) and 6(b), respectively. As illustrated in Fig. 6(a), the increase in $V_{T}$ with time for the pre-UV-irradiated SBNT-SONOS capacitor device is a result of negative charges naturally tunneling from the tunneling oxide into the SBNT-nitride charge-trapping layer of the SBNT-SONOS device before UV irradiation. As shown in Fig. 6(b), the decrease in $V_{T}$ with time for the UV-irradiated SBNT-SONOS capacitor device is a result of UV-radiation-induced negative charges naturally tunneling out from the SBNT-nitride charge-trapping layer into the tunneling oxide. For the SONOS-type nonvolatile device, it is difficult for the electrons in the trapping layer to escape to the control gate owing to the relatively larger barrier height of the thick $\mathrm{SiO}_{2}$ blocking oxide. As a result, negative charges accumulate permanently in the layers. The predicted change in $V_{T}$ after 10-year retention was extrapolated from the experimental $V_{T}-T$ curve after 1-year retention, as shown in Figs. 6(a) and 6(b). ${ }^{(6,10,11)}$ The charge-retention loss of the nonvolatile SBNT-SONOS capacitor after 10 -year retention was predicted to be below $10 \%$. 


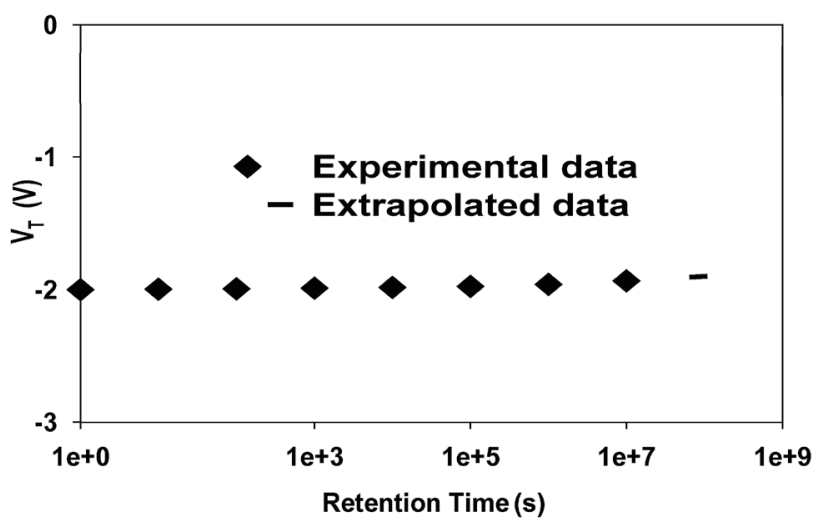

(a)

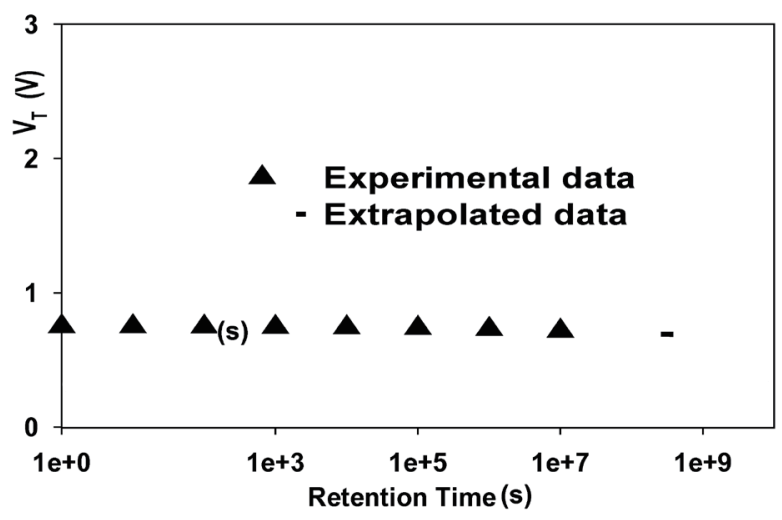

(b)

Fig. 6. $\quad V_{T}$ vs retention time for (a) SBNT-SONOS device before UV irradiation and (b) SBNT-SONOS device after UV irradiation at $100 \mathrm{~mW} \cdot \mathrm{s} / \mathrm{cm}^{2} \mathrm{TD}$ under PGV of $20 \mathrm{~V}$.

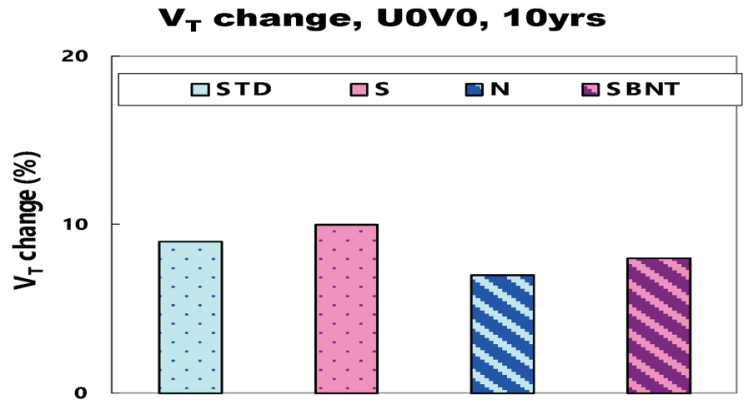

(a)

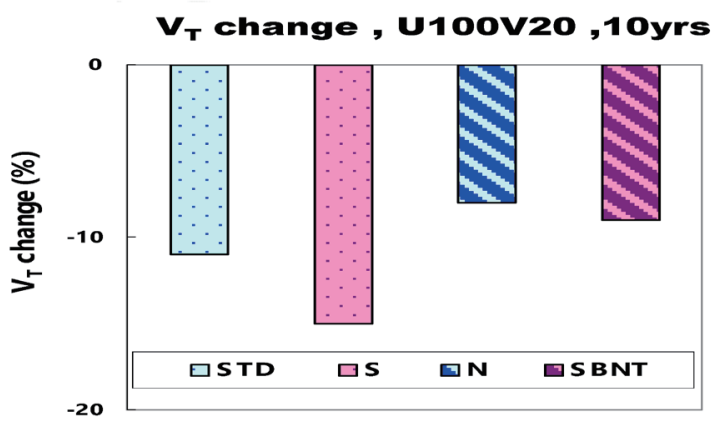

(b)

Fig. 7. (Color online) Comparisons of change in $V_{T}$ after 10-year retention for four SONOS devices (a) before UV irradiation and (b) after UV irradiation at $100 \mathrm{~mW} \cdot \mathrm{s} / \mathrm{cm}^{2} \mathrm{TD}$ under PGV of $20 \mathrm{~V}$.

Figures 7(a) and 7(b) respectively show the comparisons of change in $V_{T}$ after 10-year retention for the four type SONOS devices before and after irradiation at $100 \mathrm{~mW} \cdot \mathrm{s} / \mathrm{cm}^{2} \mathrm{UV}$ TD under a $20 \mathrm{~V}$ PGV. The loss of stored negative charges from the S-SONOS device before and after UV irradiation was greater than that from STD-SONOS, as shown in Figs. 7(a) and 7(b). As explained previously, the Si-rich nitride exhibits a high density of relatively shallow traps. A larger negative-charge loss with retention time was observed for S-SONOS owing to the relatively shallow negative-charge trap energy level $\left(E_{T A}\right)$ originating from $\mathrm{Si}-\mathrm{H}$ or $\mathrm{Si}$ dangling bonds. $^{(5,9-11)}$ In contrast, $\mathrm{N}$-rich nitride has more deep traps. A much deeper charge trap energy level $\left(E_{T A}\right)$ was observed for an N-SONOS device in previous studies. ${ }^{(5,9-11)}$ In comparison, N-rich nitride shows better reliability as a UV TD sensor than STD nitride. This result also agreed with previous studies. ${ }^{(5,9-11)}$ Note that SBNT-SONOS demonstrated better UV-induced charge-retention reliability characteristics than STD-SONOS. Because the SBNT-SONOS device has N-rich nitride on top of the charge-trapping layer, it exhibits a better charge retention reliability characteristic than the STD-MONOS device before and after UV illumination. The 
charge-retention loss of the nonvolatile SBNT-SONOS capacitor after 10-year retention is below $10 \%$. The nonvolatile SBNT-SONOS capacitor devices have very good reliability in terms of $V_{T}$ retention, even after 10 years. The UV TD information can also be permanently stored and accumulated in the nonvolatile SBNT-SONOS capacitor devices.

\section{Conclusions}

Regarding the trade-off between UV TD sensor sensitivity and reliability, the devices with bilayer nitride as the charge-trapping layer simultaneously show higher UV TD response performance and better reliability as a UV TD sensor than the devices with a single standard nitride layer. The $\mathrm{Si}-\mathrm{N}$ composition ratio is shown to significantly alter the electron- and holetrapping properties. Varying the $\mathrm{Si}-\mathrm{N}$ composition ratio from $\mathrm{N}$-rich to $\mathrm{Si}$-rich increases the electron trap density. We demonstrated that the $\mathrm{Si}-\mathrm{N}$ composition ratio also markedly changes the electron trap depth. The electron trap depth decreases as the $\mathrm{Si}-\mathrm{N}$ composition ratio is varied from N-rich to Si-rich. Higher activation energy is associated with good negative charge retention. The retention of negative charges is strongly affected by the $\mathrm{Si}-\mathrm{N}$ composition ratio and decreases as the $\mathrm{Si}-\mathrm{N}$ composition ratio becomes Si-rich. Relatively shallow negativecharge trap energy levels $E_{T A}$ originating from $\mathrm{Si}-\mathrm{H}$ or $\mathrm{Si}$ dangling bonds were observed for S-SONOS compared with STD-SONOS. Si-rich nitride has shallower traps but a higher trap density. In contrast, $\mathrm{N}$-rich nitride has deeper traps but a lower trap density. In comparison, S-rich nitride shows better performance but poorer reliability as a UV TD sensor than N-rich nitride. N-rich nitride shows better reliability but poorer performance as a UV TD sensor than Si-rich nitride. The trade-off between the UV TD response performance and the reliability requires an optimal $\mathrm{Si}-\mathrm{N}$ composition ratio to optimize the UV TD response performance and reliability. Both the UV TD response performance and the reliability are important for UV TD sensors, so we used Si-rich nitride on the bottom and N-rich nitride on the top for the trapping layer instead of a standard nitride layer for this study. Because the UV TD response performance and reliability have the same importance, the thicknesses of the N-rich nitride and Si-rich nitride layers in the bilayer stacked trapping layer were equal in this study. However, the SBNT-SONOS devices with a bilayer stacked trapping layer of Si-rich nitride on the bottom and N-rich nitride on the top simultaneously showed improvement in the performance and better reliability than the STD-SONOS devices. The bilayer stacked trapping layer with Si-rich nitride on the bottom had higher negative trap density and a greater radiation-induced buildup of negative charges, and therefore showed a better UV TD response performance as a UV TD sensor than the standard nitride. Because the SBNT-SONOS device had N-rich nitride on top of the charge-trapping layer, it exhibited better charge retention reliability than an STDMONOS device. As shown from the experimental data, the increase in the $V_{T}$ of the SBNTSONOS capacitor was nearly $3 \mathrm{~V}$, which was twice that of the STD-SONOS capacitor after UV irradiation at $100 \mathrm{~mW} \cdot \mathrm{s} / \mathrm{cm}^{2}$ under a PGV of $20 \mathrm{~V}$. The UV-radiation-induced change in the $V_{T}$ of the SBNT-SONOS capacitor also correlated with UV irradiation up to $100 \mathrm{~mW} \cdot \mathrm{s} / \mathrm{cm}^{2}$ TD. However, the $V_{T}$ of the SBNT-SONOS capacitor did not change significantly after only UV irradiation at $100 \mathrm{~mW} \cdot \mathrm{s} / \mathrm{cm}^{2} \mathrm{TD}$ (without PGV) or with only a PGV of $20 \mathrm{~V}$ (without UV 
irradiation). The $100 \mathrm{~mW} \cdot \mathrm{s} / \mathrm{cm}^{2} \mathrm{UV}$-induced charge-retention loss of the nonvolatile SBNTSONOS capacitor after 10 -year retention was below $10 \%$. The results obtained in this study have demonstrated that UV TD information can be permanently stored and accumulated in nonvolatile SBNT-SONOS capacitor devices.

\section{Acknowledgments}

The authors thank the National Nano Device Laboratories (NDL), National Tsing Hua University (NTHU), and National Chiao Tung University (NCTU) for providing the instruments needed for wafer fabrication and testing. This study was funded in part by the National Science Council (NSC).

\section{References}

1 W. C. Hsieh, H. T. Lee, F. C. Jong, and S. C. Wu: Proc. 2013 Int. Conf. Advanced Infocomm Technology (IEEE, 2013) 163.

2 F. C. Jong, W. C. Hsieh, H. T. Lee, and S. C. Wu: Sens. Mater. 8 (2018) 1831.

3 A. Abraham, N. Nicola, N. Diana, and M. Curiel: Key Eng. Mater. 605 (2014) 380.

4 W. C. Hsieh, H. T. Lee, F. C. Jong, and S. C. Wu: Sens. Mater. 5 (2016) 577.

5 W. C. Hsieh, H. T. Lee, F. C. Jong, and S. C. Wu: Sens. Mater. 9 (2016) 1023.

6 W. C. Hsieh, H. T. Lee, F. C. Jong, and S. C. Wu: Sens. Mater. 7 (2017) 969.

7 T. R. Oldham and F. B. McLean: IEEE Trans. Nucl. Sci. 50 (2003) 483.

8 Y. Cheng, M. Ding, X. Wu, X. Liu, and K. Wu: Proc. 2013 Int. Conf. Solid Dielectrics (IEEE, 2013) 764.

9 J. P. Colonna, E. Nowak, and G. Molas: Proc. 27th Int. Conf. Microelectronics (MIEL) (2010) 1.

10 C. Sandhya, A. B. Oak, N. Chattar, A. S. Joshi, and U. Ganguly: IEEE Trans. Electron Devices 56 (2009) 3123.

11 C. Sandhya, U. Ganguly, K. K. Singh, P. K. Singh, C. Olsen, S. M. Seutter, R. Hung, G. Conti, K. Ahmed, N. Krishna, J. Vasi, and S. Mahapatra: Proc. 2008 IEEE Int. Reliability Physics Symp. (IEEE, 2008) 406.

\section{About the Authors}

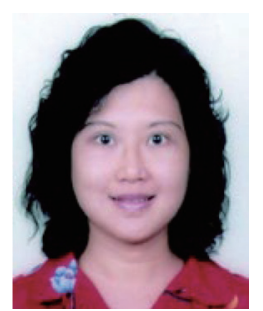

Wen Ching Hsieh received her B.S. degree in physics from National Cheng Kung University (NCKU), Tainan, Taiwan, in 1986 and her Ph.D. degree from the Institute of Nuclear Science of National Tsing Hua University (NTHU), Hsinchu, Taiwan, in 1994. From 1994 to 1995, she was with Winbond Electronics Corporation, Hsinchu, Taiwan, as a research engineer in the memory R\&D division, where she worked on the development of flash EEPROM memory devices. In 1996, she joined the memory R\&D division of Taiwan Semiconductor Manufacturing Company, Hsinchu, Taiwan, as a technical manager, where she worked on the simulation and characterization of flash memory devices. Since 2000, she has been with the department of Opto-Electronic System Engineering in Minghsin University of Science and Technology (MUST), Hsinchu, Taiwan, where she is currently an associate professor. She is the supervisor of the semiconductor photosensor nanodevice laboratory at MUST. Her research interests are radiation/photosensor devices using advanced NVM device processes including high-k gate dielectrics and metal gates. She has published over 50 technical papers. 
Fuh-Cheng Jong received his B.S. degree in electrical engineer from Chinese Culture University (CCU), Taipei, Taiwan, in 1987 and his Ph.D. degree from the Institute of Electronics Engineering of National Chiao Tung University (NCTU), Hsinchu, Taiwan, in 1997. From 1994 to 1996, he was with Winbond Electronics Corporation, Hsinchu, Taiwan, as a research engineer in the memory product division, where he worked on the development of flash EEPROM memory devices. In 2000, he joined the memory R\&D division of Macronix, Hsinchu, Taiwan, as a technical manager, where he worked on the characterization of flash memory devices. Since 2002, he has been with the Department of Electronic Engineering in Southern Taiwan University, Tainan, Taiwan, where he is currently an assistant professor. 